\title{
Ten CoRoT eclipsing binaries: Photometric solutions
}

\author{
S.C. Maciel *, Y.F.M. Osorio, J.R. De Medeiros \\ Departamento de Física, Universidade Federal do Rio Grande do Norte, 59072-920 Natal, RN, Brazil
}

\section{A R T I C L E I N F O}

\section{Article history:}

Received 28 June 2010

Received in revised form 20 July 2010

Accepted 21 July 2010

Available online 24 July 2010

Communicated by P.S. Conti

\section{Keywords:}

Star

CoRoT

Eclipsing binaries

Fundamental parameters

\begin{abstract}
A B S T R A C T
Ten eclipsing binaries, identified in the scope of the CoRoT Space Mission, were selected for analysis. The photometric light curves were processed and analyzed, resulting in the first study of eclipsing binary candidates with their possible photometric solution, in the context of the above-mentioned Space Mission. The selected targets are detached and overcontact systems, for which we computed 2MASS temperatures, in addition to different physical parameters, including orbital period, orbit inclination angle, and temperatures, radius and luminosity ratios. This study reveals a large diversity of eclipsing binary systems obtained from the CoRoT data.
\end{abstract}

(c) 2010 Elsevier B.V. All rights reserved.

\section{Introduction}

CoRoT (Convection, Rotation and planetary Transits) is a photometric space experiment, launched on December 27th 2006 in an inertial polar orbit at an altitude of $897 \mathrm{~km}$. It is devoted to asteroseismology and the search for extrasolar planets using the transit method (Baglin et al., 2006). The instrument is fed by a $27-\mathrm{cm}$ diameter telescope. During each run, it simultaneously provides light curves (variations in stellar flux with time) from 10 bright stars $\left(5.5<\mathrm{m}_{\mathrm{V}}<9.5\right)$ dedicated to seismic studies, while 12,000 fainter stars $\left(11.5 \mathrm{~m}_{\mathrm{V}}<15.5\right)$ are monitored to search for transits caused by planets. Exhibiting a high-precision photometry of 100-600 ppm, combined with long time coverage of target areas, CoRoT also offers a unique possibility for the detection and study of eclipsing binary systems. In this context, we analyzed one thousand light curves from the CoRoT public data archive, ${ }^{1}$ searching for transit signature of binary systems, from which we identified 10 binary eclipsing systems illustrating an interesting diversity of stellar binary families. With this preliminary study we also aim to alert the community to the large variety of binaries obtained from the CoRoT Space Mission data, which offer numerous possibilities for enlarging our understanding of the behavior and evolution of stellar binary systems.

\footnotetext{
The CoRoT space mission was developed and is operated by the French space agency CNES, with the participation of ESAs RSSD and Science Programmes, Austria, Belgium, Brazil, Germany, and Spain.

* Corresponding author. Fax: +55 08432153791.

E-mail address: saulo@dfte.ufrn.br (S.C. Maciel).

${ }^{1}$ http://idoc-corot.ias.u-psud.fr.
}

\section{General analysis of the Corot targets}

The basic parameters for our sample of 10 binary systems are displayed in Table 1 . In addition to the star CoRoT code and coordinates (RA and Dec), Table 1 also shows the spectral type (Sp) and luminosity class (LC) of each system, extracted from CoRoT data base. Star ID 102622874, ID 102673160 and ID 102738809 were for the first time identified as eclipsing binaries in the scope of the present study, whereas ID 102764398, ID 102773399, ID 102794063, ID 102806409 and ID 102961901 were identified by Carpano et al. (2009) also on the basis of CoRoT data. Star ID 102750270 (Gu Mon) and ID 102940723 (V515Mon) are already known in the literature as eclipsing binaries, a status confirmed by CoRoT observations. It should be underlined that all the light curves analyzed in the present study come from the first long run observed by CoroT, namely the IRa01, toward the galactic anticenter.

The study of eclipsing binary systems is based on the changes of light intensity from eclipsing binary stars that occur when one of the star transits in front of the other relative to the observer (Kopal, 1975). The 10 light curves were reduced with a standard pipeline developed at Laboratoire d'Astrophysique de Marseille (LAM) by the CoRoT Community (Samadi et al., 2007; De Oliveira Fialho et al., 2007). Different artifacts such as linear trends and discontinuities were removed from the original data. Due to the large PSF (point spread function) of CoRoT, a false binary may be found (Barge et al., 2008; Drummond et al., 2008). It should be underscored that an important detail to confirm the eclipse is to observe the eclipsing signature in the three photometric bands of the CoRoT mask separately. Such an aspect is observed for all the stars of the sample. 
The orbital period of each binary system, listed in Table 2, was calculated using the DCDFT method (date-compensated discrete Fourier transform). In order to derive the physical parameters from the light curve, we used the PHOEBE programme (Prs a and Zwitter, 2005) which functions based on the Wilson-Devinney algorithm (Wilson and Devinney, 1971). This programme was expanded to include flux computation in the CoRoT passbands
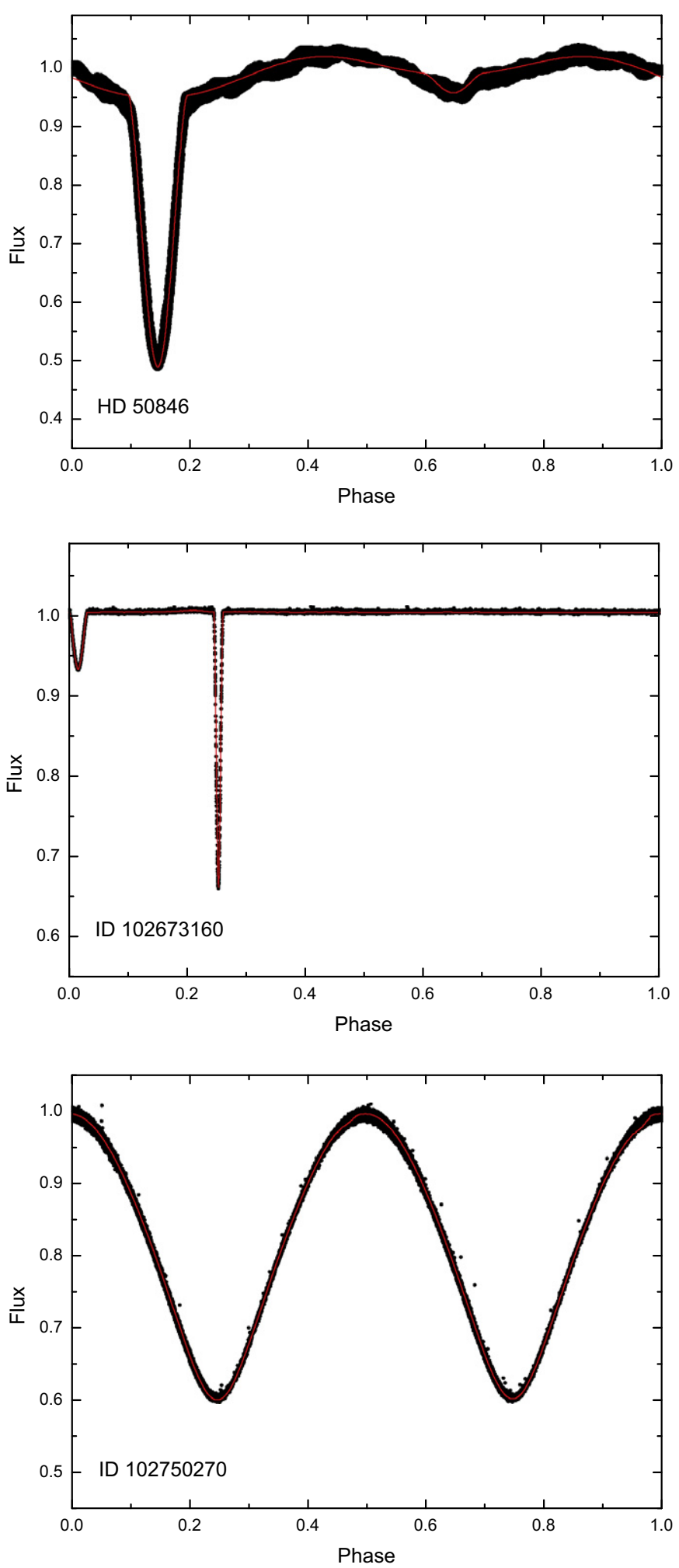

for both seismology and exoplanet fields (Maceroni et al., 2009). To find the best fit for these systems we used two types of Phoebe models for different types of binary systems, detached binary (ID 102673160, ID 102738809, ID 102764398, ID 102806409 and ID 102940723) over contact binary not in thermal contact (ID 102622874, ID 102750270, ID 102773399, ID 102794063 and ID 102961901). For the bolometric albedo and gravity darkening we
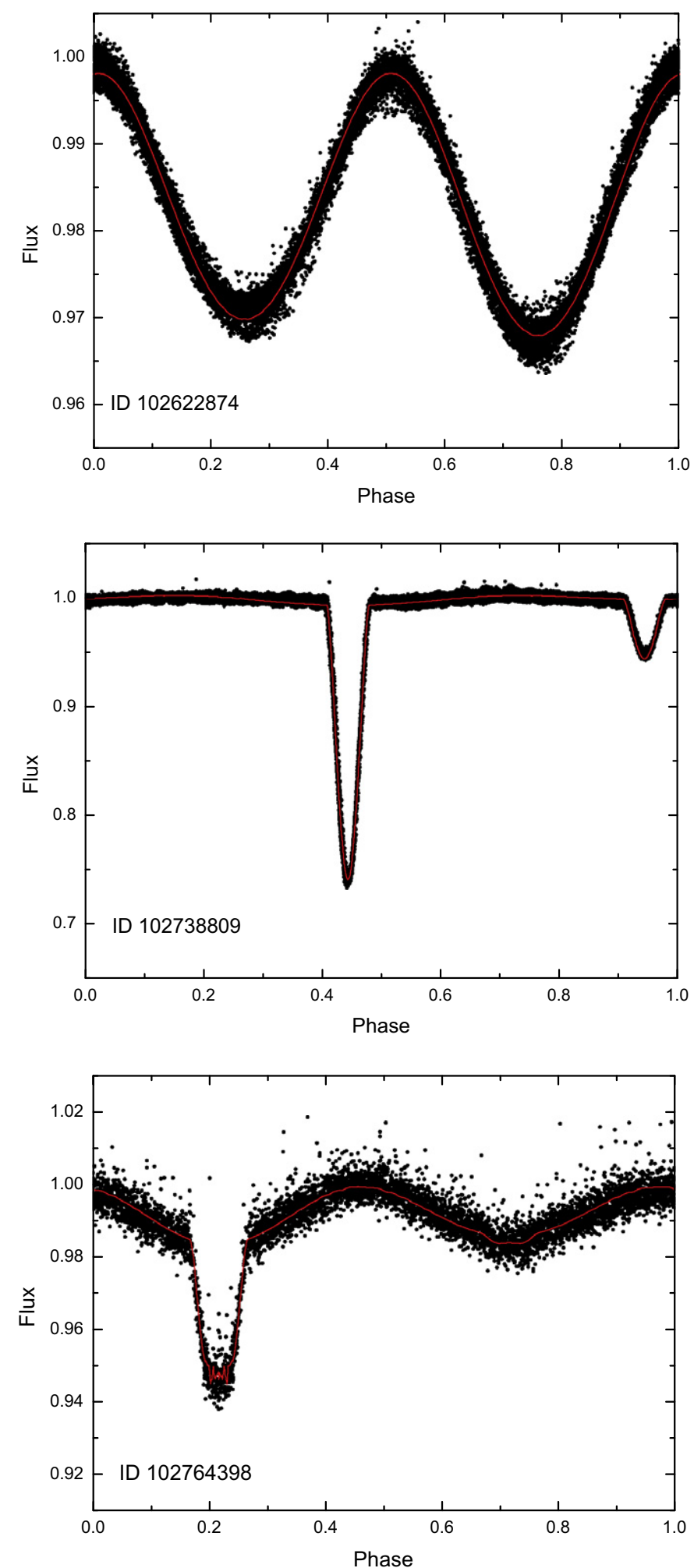

Fig. 1. The phase diagram of HD 50846 and CoRoT targets ID 102622874, ID 102673160, ID 102738809, ID 102750270, ID 102764398. 
used the theoretical values for convective atmospheres, i.e. $A_{1}=A_{2}=0.5, G_{1}=G_{2}=0.32$ (Lucy, 1968) and these values were kept fixed. Specific limb-darkening coefficients were computed from Sing's table (Sing, 2010) using the quadratic law, which provides two $x_{1}\left(x_{2}\right)$ and $y_{1}\left(y_{2}\right)$ coefficients per star. The temperature of the primary stars $\left(T_{1}\right)$ was kept fixed and calibrated from the color indices $(J-K)$ provided from the 2MASS catalog, using the calibrations of Alonso et al. (1999).
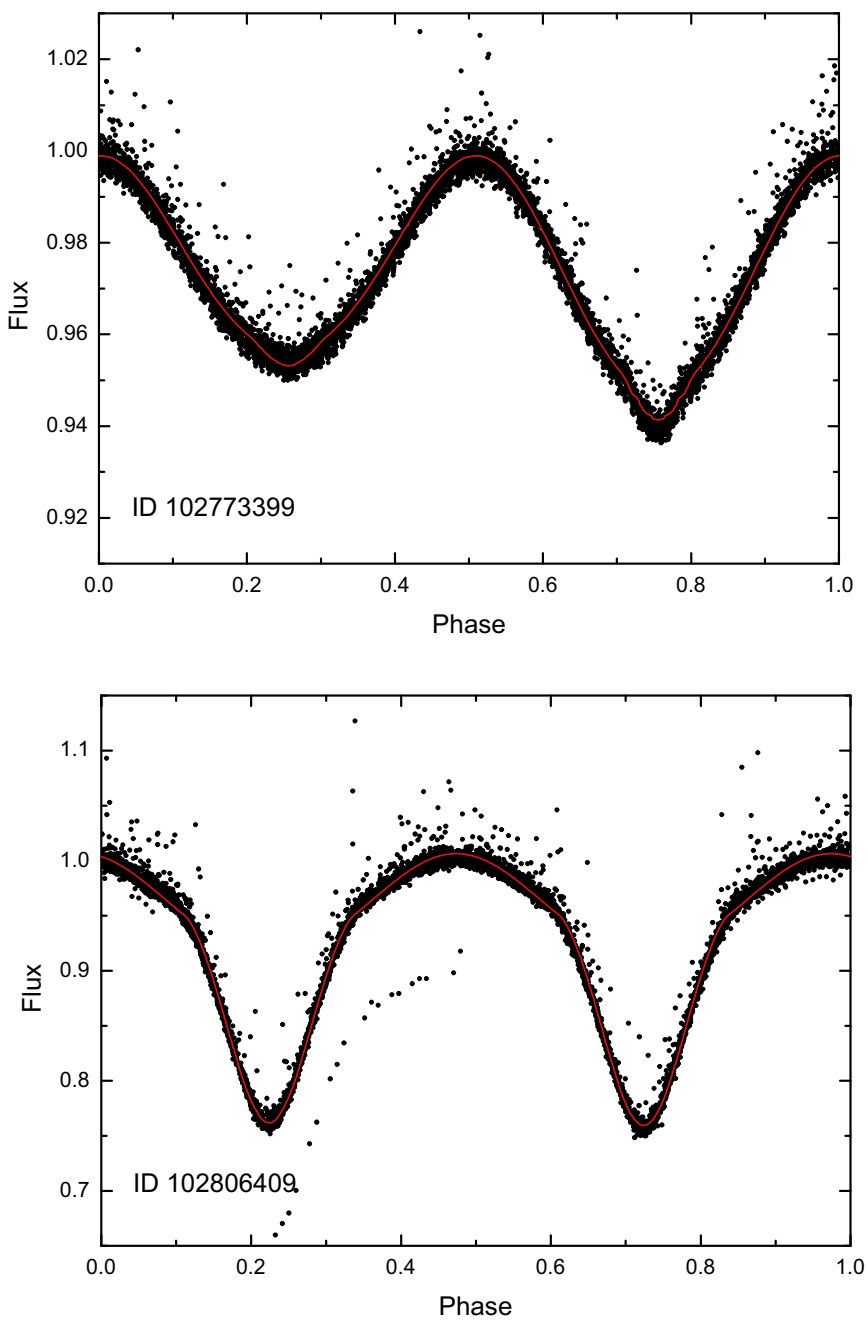

All the stars have circular orbits except CoRoT ID 102673160 , which has a very eccentric orbit with $\mathrm{e}=0.60968$ and argument of periastron $\omega=0.91399 \mathrm{rad}$. The parameters adjusted in the minimizations were inclination $i$, temperature of secondary star $T_{2}$, primary passband luminosity $L_{i}$ and surface potentials $\Omega_{1}$ and $\Omega_{2}$. The mass ratio was set at 1.0 and when the adjustment of these parameters did not provide further improvement, we included mass ratio in the minimization calculation. For CoRoT ID 102673160 we first
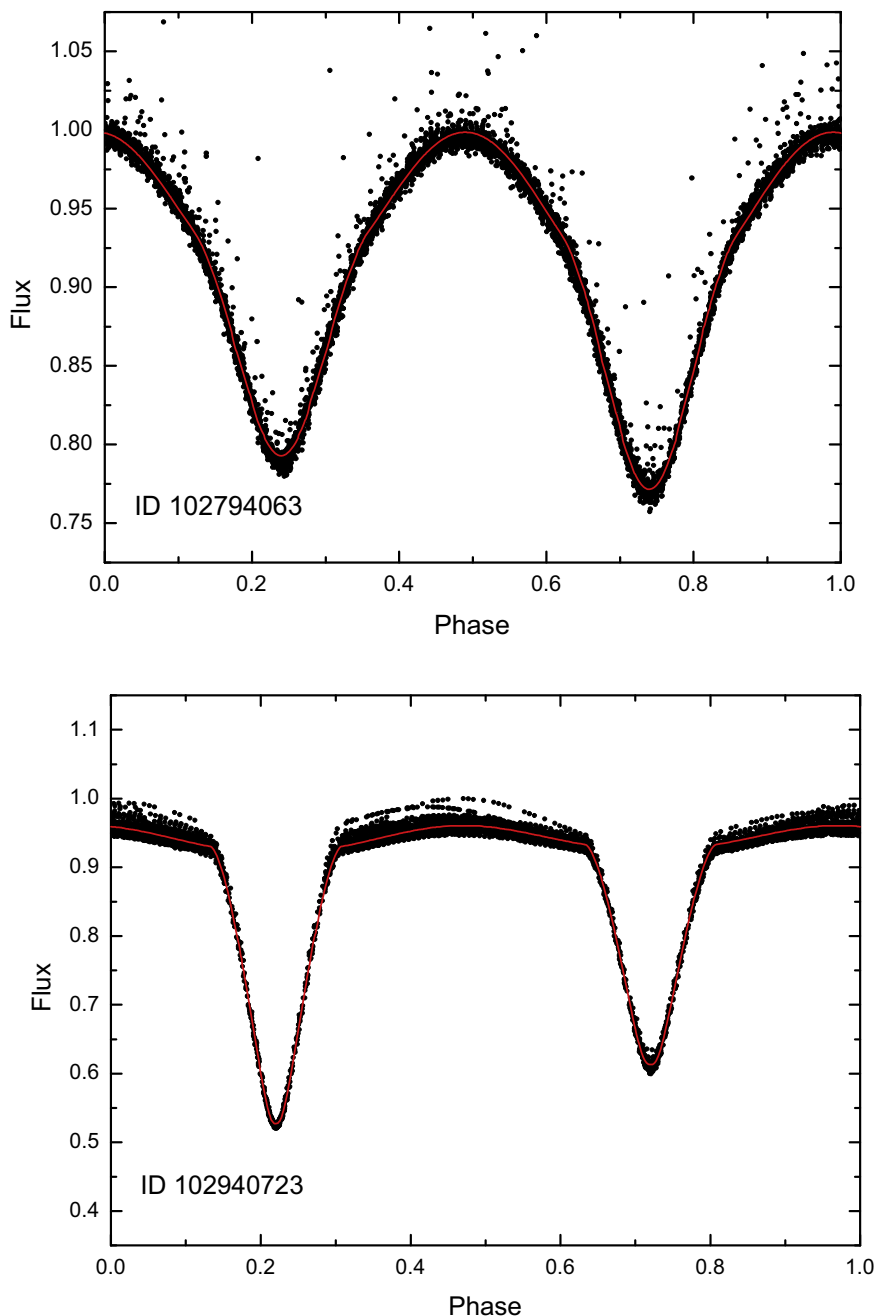

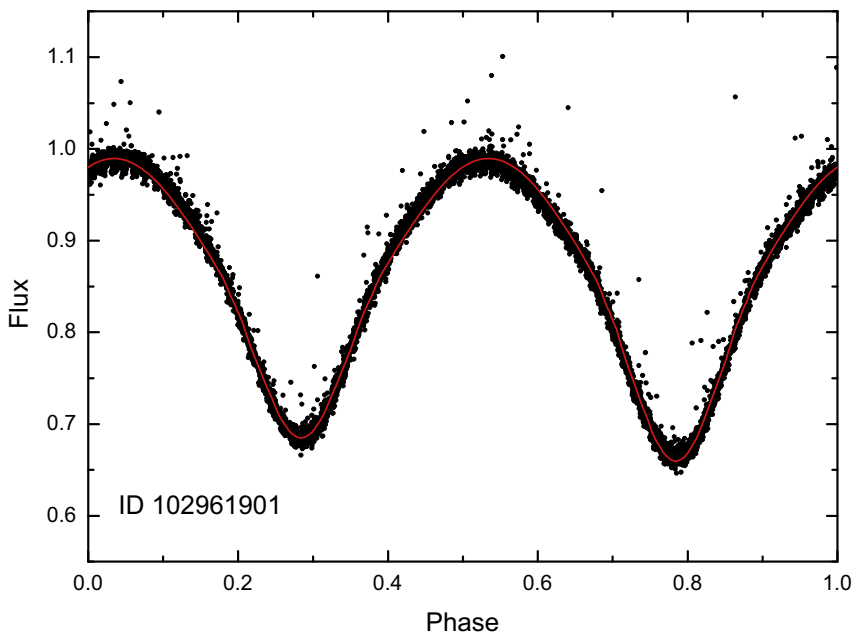

Fig. 2. The phase diagram of CoRoT targets ID 102773399, ID 102794063, ID 102806409, ID 102940723 and ID 102961901. 
Table 1

Basic information about the analyzed systems.

\begin{tabular}{lllllll}
\hline COROT ID & RA & Dec & Sp & LC & $(J-K)$ & $T_{1}$ \\
\hline 102622874 & 064158.73 & -000438.86 & F7 & III & 0.240 & 6360 \\
102673160 & 064305.43 & -003440.08 & F8 & IV & 0.281 & 6095 \\
102738809 & 064431.53 & +004955.67 & F2 & III & 0.247 & 6313 \\
102750270 & 064446.85 & +001318.44 & F2 & II & 0.130 & 7221 \\
$\quad$ (Gu Mon) & & & & & & \\
102764398 & 064505.96 & -024840.36 & F8 & IV & 0.444 & 5257 \\
102773399 & 064518.13 & -005700.40 & G1 & V & 0.412 & 5399 \\
102794063 & 064546.02 & -024833.80 & G8 & V & 0.584 & 4729 \\
102806409 & 064610.00 & -030855.46 & A0 & V & 0.412 & 5399 \\
102940723 & 064921.14 & -020648.82 & A5 & IV & 0.249 & 6299 \\
$\quad($ V515 Mon) & & & & & & \\
102961901 & 064946.29 & -020105.81 & G2 & IV & 0.583 & 4732 \\
\hline
\end{tabular}

Table 2

Light-curve parameters of the individual systems.

\begin{tabular}{lrllllr}
\hline COROT ID & \multicolumn{1}{c}{$P$} & $i$ & $q$ & $T_{1} / T_{2}$ & $R_{1} / R_{2}$ & \multicolumn{1}{c}{$L_{1} / L_{2}$} \\
\hline 102622874 & 4.150 & 44.698 & 0.636 & 1.04 & 1.29 & 2.00 \\
102673160 & 28.918 & 86.713 & 0.856 & 1.00 & 1.03 & 1.05 \\
102738809 & 2.035 & 79.325 & 1.009 & 1.36 & 0.51 & 1.18 \\
102750270 & 0.897 & 72.137 & 0.900 & 1.00 & 1.04 & 1.06 \\
102764398 & 0.927 & 79.269 & 0.226 & 1.77 & 5.28 & 898.12 \\
102773399 & 0.606 & 50.626 & 0.286 & 1.18 & 1.92 & 8.96 \\
102794063 & 0.382 & 63.949 & 1.000 & 1.04 & 1.00 & 1.20 \\
102806409 & 0.647 & 68.818 & 0.938 & 1.00 & 1.02 & 1.05 \\
102940723 & 0.874 & 86.607 & 1.000 & 1.05 & 1.28 & 2.10 \\
102961901 & 0.421 & 68.730 & 1.000 & 1.03 & 1.00 & 1.17
\end{tabular}

Table 3

Light-curve parameters of the individual systems (continuation).

\begin{tabular}{|c|c|c|c|c|c|c|c|}
\hline COROT ID & Phsh & $\Omega_{1}$ & $\Omega_{2}$ & $x_{1}$ & $x_{2}$ & $y_{1}$ & $y_{2}$ \\
\hline 102622874 & -0.241 & 3.619 & 3.619 & 0.359025 & 0.382100 & 0.282404 & 0.267984 \\
\hline 102673160 & +0.337 & 23.365 & 21.271 & 0.382203 & 0.380705 & 0.267842 & 0.268540 \\
\hline 102738809 & +0.443 & 11.272 & 6.343 & 0.361451 & 0.636541 & 0.282813 & 0.085011 \\
\hline 102750270 & -0.254 & 3.181 & 3.181 & 0.294220 & 0.293424 & 0.327532 & 0.328204 \\
\hline 102764398 & +0.216 & 3.623 & 5.401 & 0.529083 & 0.314593 & 0.168507 & 0.388917 \\
\hline 102773399 & -0.244 & 2.606 & 2.606 & 0.502402 & 0.609873 & 0.188159 & 0.104685 \\
\hline 102794063 & -0.260 & 3.863 & 3.863 & 0.615920 & 0.613206 & 0.099535 & 0.104410 \\
\hline 102806409 & -0.276 & 3.894 & 3.849 & 0.502250 & 0.505260 & 0.188210 & 0.185990 \\
\hline 102940723 & +0.220 & 4.488 & 5.419 & 0.364129 & 0.407964 & 0.280646 & 0.254073 \\
\hline 102961901 & -0.216 & 3.709 & 3.709 & 0.617404 & 0.621043 & 0.098450 & 0.097288 \\
\hline
\end{tabular}

computed the eccentricity and longitude of the periastron. The final fit for each star is displayed in Figs. 1 and 2, which shows the phase diagrams represented by black points, namely the normalized flux versus phase for all the stars of the present working sample. In these figures the red line represents the PHOEBE model. From the analysis of the light curve we can obtain the following quantities: Period (P), temperature ratio $T_{1} / T_{2}$, inclination $i$, Kopal's modified potentials $\Omega_{1}$ and $\Omega_{2}$ and radii ratio $R_{1} / R_{2}$. All these values are also listed in Tables 2 and 3, including a phase shift (Phsh) of the primary eclipsing.

To check the reliability of the present procedure, we computed the physical parameters from the CoRoT light curve of HD 50846 (Desmet et al., 2010). All the values obtained by these authors are reproduced in our procedure. The phase diagram for this binary system is displayed in Fig. 1.

\section{Discussion and conclusions}

We analyzed light curves of 10 eclipsing binaries identified in the scope of the CoRoT Space Mission. In addition to the orbital period, we computed the possible photometric solution for each eclipsing binary system. In these systems the temperature, luminosity and radius of the primary component are higher than the values obtained for the secondary, except for ID 102738809, where the secondary radius is higher than the primary. Of course, for a complete solution of each binary system, which includes the absolute determination of all parameters for each star and a full orbital solution, it is essential to combine photometric and spectroscopic informations. Nevertheless, one of the major goals of the present study is to alert the community to the large variety of binaries obtained from the CoRoT Space Mission data. For instance, one can observe from the phase diagram that our sample consists of several subtypes of binary systems such as the Algol type (ID 102673160, ID 102738809 and ID 102940723), Beta Lyrae type (ID 102764398,
ID 102773399 and ID 102806409) and Wuma type (ID 102622874, ID 102750270, ID 102794063 and ID 102961901).

\section{Acknowledgments}

Research activities at the Stellar Board of the Universidade Federal do Rio Grande do Norte are supported by continuous grants of CNPq and FAPERN Brazilian agencies. S.C. Maciel acknowledges a PhD felowship of CAPES Brazilian Agency. We thank Dr. I.C. Leão for the help with the programs used in this work and C. Cortes and Lopes C.E.F. for the discussions.

\section{References}

Alonso, A., Arribas, S., Martnez-Roger, C., 1999. A\&AS 140, 261.

Baglin, A., Auvergne, M., Barge, P., Deleuil, M., Catala, C., Michel, E., Weiss, W.The COROT Team, 2006. ESASP 1306, 33.

Barge, P., Baglin, A., Auvergne, M.The CoRoT Team, 2008. IAUS 249, 3.

Carpano, S., Cabrera, J., Alonso, R., Barge, P., Aigrain, S., Almenara, J.-M., Bord, P. Bouchy, F., Carone, L., Deeg, H.J., de La Reza, R., Deleuil, M., Dvorak, R., Erikson, A., Fressin, F., Fridlund, M., Gondoin, P., Guillot, T., Hatzes, A., Jorda, L., Lammer, H., Lger, A., Llebaria, A., Magain, P., Moutou, C., Ofir, A., Ollivier, M., JanotPacheco, E., Ptzold, M., Pont, F., Queloz, D., Rauer, H., Rgulo, C., Renner, S., Rouan, D., Samuel, B., Schneider, J., Wuchterl, G., 2009. A\&A 506, 491.

De Oliveira Fialho, F., Lapeyrere, V., Auvergne, M., Drummond, R., Vandenbussche, B., Aerts, C. Kuschnig, R., Matthews, J.M., 2007. PASP 119, 337.

Desmet, M., Frmat, Y., Baudin, F., Harmanec, P., Lampens, P., Janot Pacheco, E., Briquet, M., Degroote, P., Neiner, C., Mathias, P., Poretti, E., Rainer, M., Uytterhoeven, K., Amado, P.J., Valtier, J.-C., Prs a, A., Maceroni, C., Aerts, C., 2010. MNRAS 401, 418 .

Drummond, R., Lapeyrere, V., Auvergne, M., Vandenbussche, B., Aerts, C., Samadi, R., Costa, J.E.S., 2008. A\&A 487, 1209.

Kopal, Z., 1975. Ap\&SS 34, 431.

Lucy, L.B., 1968. ApJ 151, 1123.

Maceroni, C., Montalbn, J., Michel, E., Harmanec, P., Prsa, A., Briquet, M., Niemczura, E., Morel, T., Ladjal, D., Auvergne, M., Baglin, A., Baudin, F., Catala, C., Samadi, R., Aerts, C., 2009. A\&A 508, 1375.

Prs a, A., Zwitter, T., 2005. ApJ 628, 426.

Samadi, R., Fialho, F., Costa, J.E.S., Drummond, R., Pinheiro Da Silva, L., Baudin, F., Boumier, P. Jorda, L., 2007. In: The Corot Book. ESA-SP 1306 (Chapter V.5)

Sing, D.K., 2010. A\&A 510, 21

Wilson, R.E., Devinney, E.J., 1971. ApJ 166, 605 